\title{
Images in Surgical Radiology: An Unusual Case of Acute Appendicitis Within a Femoral Hernia
}

\author{
Emma Aitken - Bryce Renwick • \\ Sheila Kate Fitzgerald • Birinder Singh • \\ Joseph Cumming
}

Received: 29 September 2010 /Accepted: 4 March 2012 /Published online: 16 March 2012

(C) Association of Surgeons of India 2012

\begin{abstract}
We present a rare case of acute appendicitis within a femoral hernia, which was diagnosed preoperatively on CT scan.
\end{abstract}

Keywords Appendicitis · Femoral hernia

\section{Introduction}

De Garengeot first described the presence of the appendix within a femoral hernia in 1731. It is a rare occurrence found in less than $1 \%$ of femoral hernias [1]. Even less common is the occurence of appendicitis within a hernia occur (thought to be the consequence of luminal obstruction) $[1,2]$.

\section{Case Report}

We report an unusual case of a 74-year-old woman who presented with a 2-day history of painful swelling in her right groin. She had borderline pyrexia $\left(37.8^{\circ} \mathrm{C}\right)$ and a tachycardia. Clinical examination revealed a tender, irreducible swelling below and lateral to the pubic tubercle with edematous, erythematous overlying skin. CT scan confirmed the clinical diagnosis of an incarcerated femoral hernia; however, it also raised the suspicion of acute appendicitis of an appendix within the hernia (Fig. 1). In theater the CT findings were confirmed. The femoral hernia was approached via a low incision. The sac was opened confirming an appendix with necrotic tip within the hernial sac. The appendix was reduced back into the abdominal

E. Aitken $(\bowtie) \cdot$ B. Renwick $\cdot$ S. K. Fitzgerald $\cdot$ B. Singh •

J. Cumming

Department of General Surgery,

Monklands District General Hospital,

Airdrie, Scotland, UK

e-mail: emmaaitken@doctors.net.uk cavity. A standard appendicectomy was then performed via a Lanz incision prior to sutured repair of the defect at the femoral canal. The patient made a good recovery and was discharged home on the 4th postoperative day. Histopathology subsequently confirmed transmural appendicitis.

To date there are only approximately 60 cases of acute appendicitis within a femoral hernia described in the literature [2]. Preoperative diagnosis is rarely made, and misdiagnosis either as an Amyand's hernia or a simple incarcerated femoral hernia is conmmon [3]. We highlight this atypical presentation of acute appendicitis and advocate the role of preoperative CT scanning in making the diagnosis.

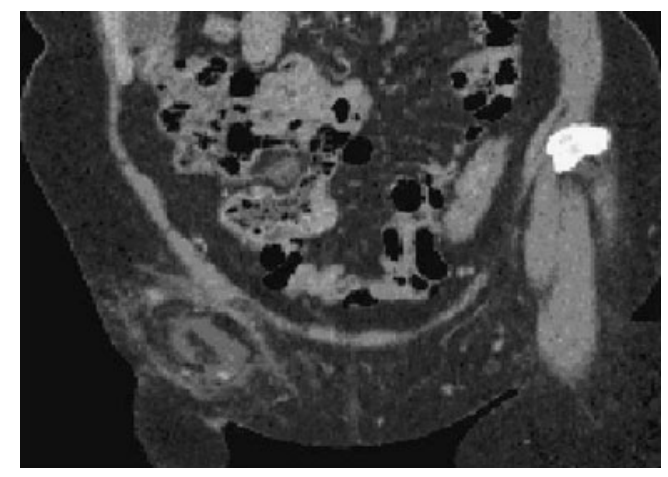

Fig. 1 Contrast-enhanced CT scan demonstrating an acutely inflamed, edematous appendix, herniating through the femoral canal

\section{References}

1. Akopian G, Alexander M (2005) De Garengeot hernia: appendicitis within a femoral hernia. Am Surg 71:526

2. Sharma H, Jha PK, Shekhawat NS et al (2007) De Garengeot hernia: an analysis of our experience. Hernia 11:235

3. Chung A, Goel A (2009) De Garengeot's hernia. NEJM 361:e18 